\title{
The lymphocyte-monocyte ratio predicts tumor response and survival in patients with locally advanced esophageal cancer who received definitive chemoradiotherapy
}

\author{
This article was published in the following Dove Press journal: \\ OncoTargets and Therapy \\ 14 February 2017 \\ Number of times this article has been viewed
}

\author{
Xuemei Liu',2,* \\ Minghuan $\mathrm{Li}^{2}, *$ \\ Fen Zhao ${ }^{2}$ \\ Yingming Zhu ${ }^{2}$ \\ Yijun Luo 2 \\ Li Kong ${ }^{2}$ \\ Hui Zhu \\ Yan Zhang ${ }^{2}$ \\ Fang $\mathrm{Shi}^{2}$ \\ Jinming $\mathrm{Yu}^{2}$ \\ 'School of Medicine and Life Sciences, \\ University of Jinan-Shandong Academy \\ of Medical Sciences, ${ }^{2}$ Department of \\ Radiation Oncology, Shandong Cancer \\ Hospital and Institute, Jinan, Shandong, \\ People's Republic of China \\ *These authors contributed equally \\ to this work
}

Correspondence: Jinming Yu

Department of Radiation Oncology, Shandong Cancer Hospital and Institute, 440 Jiyan Road, Jinan 250I I7, Shandong, People's Republic of China

Tel +8653I 87984729

Fax +86 53। 87984079

Email sdyujinming@163.com
Background: The lymphocyte-monocyte ratio (LMR), a simple biomarker that can reflect the antitumor immune response of the host, has been associated with patient prognosis in several solid tumors. The aim of this study was to evaluate whether LMR can predict clinical tumor response and prognosis in patients with locally advanced esophageal squamous cell carcinoma (ESCC) who received definitive chemoradiotherapy (CRT).

Patients and methods: A total of 162 advanced ESCC patients treated at our institution between January 2012 and December 2013 were retrospectively recruited for analysis. Patients were treated with a platinum-based bimodal cytotoxic drug chemotherapy and concurrent radiation therapy. The LMR was calculated from blood counts in samples collected prior to treatment initiation. The predictive value of LMR for clinical tumor response and prognosis was examined.

Results: The LMR before CRT was significantly higher in 48 patients who achieved clinical complete response (CR) compared to that in patients who did not achieve clinical CR ( $4.89 \pm 1.17$ vs $3.87 \pm 1.29, P<0.001)$. Compared to their matched counterparts, patients in the high LMR group (LMR $>4.02)$ showed a good clinical tumor response $(P<0.05)$. A significant independent association between a high pretreatment LMR and better outcomes was identified in a multivariate analysis for progression-free survival (PFS; hazard ratio $[\mathrm{HR}]=2.17 ; P<0.001$ ) and overall survival (OS; HR=2.02; $P=0.002$ ).

Conclusion: In ESCC patients, a high LMR before treatment, which indicates a robust host immune system, is associated with both a good clinical tumor response after definitive CRT and favorable prognosis.

Keywords: esophageal squamous cell carcinoma, lymphocyte-monocyte ratio, definitive chemoradiotherapy, tumor response, prognosis

\section{Introduction}

Recent estimations rank esophageal cancer (EC) as the 6th leading cause of cancer-related deaths worldwide. ${ }^{1}$ The prognosis is extremely poor, with only a $21 \%$ 5 -year overall survival (OS) rate and frequent local recurrence or distant metastases. ${ }^{2}$ The major pathological type of EC in East Asia is esophageal squamous cell carcinoma (ESCC), whereas adenocarcinoma is predominant in Western countries. ${ }^{1}$ More than $60 \%$ of newly diagnosed EC patients present either locally advanced or metastatic disease. ${ }^{3}$ For these patients, a chemoradiotherapy (CRT)-based multidisciplinary treatment may be the only approach to achieve a definitive cure instead 
of an esophagectomy. For patients undergoing definitive CRT, 50.4 Gy is the accepted standard radiation dose based on randomized data from Europe and North America. ${ }^{4,5}$ Although radiation dose escalation has failed to improve either local control or survival, a 60 Gy dose is more popular in Asian countries where squamous cell carcinoma is the predominant histological subtype. ${ }^{6,7}$ However, for patients with local advanced ESCC, only a small percentage will achieve a major or complete response (CR) after radiation and chemotherapy. ${ }^{5-7}$

The tumor-node-metastasis (TNM) staging system is thought to be an effective predictor in most prognostic models. ${ }^{8}$ However, the conventional TNM staging system is unable to provide a precise prediction of prognosis in clinical practice, which highlights the necessity of identifying new parameters to complement the TNM stage and assist in improving individualized treatment. Currently, it is widely accepted that inflammatory cells, which are present in the tumor microenvironment, ${ }^{9-11}$ and the tumor immune response, which is activated by ionizing radiation, ${ }^{12}$ significantly affect tumor development. This tumor-generated inflammatory response may result in an increased propensity for apoptosis, angiogenesis, and DNA damage by upregulating cytokines and inflammatory mediators. ${ }^{13-15}$ The lymphocytemonocyte ratio (LMR) is thought to reflect the degree of systemic inflammation and has been touted as an important prognostic indicator in non-small-cell lung cancer, ${ }^{16}$ pancreatic adenocarcinoma, ${ }^{17}$ melanoma, ${ }^{18}$ and nasopharyngeal carcinoma. ${ }^{19}$ The prognostic value of LMR has expanded to include some solid tumors based on a systematic review and meta-analysis. ${ }^{20}$ All these findings focused on the predictive value of LMR for long-term survival, but few studies compared the LMR with therapy responsiveness, which is also critical in determining clinical treatment methods. Therefore, we conducted this study to explore the role of the LMR before cancer treatment in predicting the tumor response and outcomes of patients with locally advanced ESCC who received definitive $\mathrm{CRT}$.

\section{Patients and methods}

\section{Patients}

We retrospectively identified and analyzed the records of ESCC patients treated with definitive CRT between January 2012 and December 2013 at the Shandong Cancer Hospital and Institute. Patients were included if they had an Eastern Cooperative Oncology Group (ECOG) performance status between 0 and 2; histologically confirmed squamous cell carcinoma of the esophagus; and fulfilled the following criteria: 1) T3-4 stage (based on the 7th edition of the
American Joint Committee on Cancer [AJCC] guidelines) as determined by either endoscopic ultrasonography (EUS) and contrast-enhanced computed tomography (CT) or positron emission tomography (PET)-CT; 2) no distant node or organ metastasis (M0); 3) no bleeding, perforations, or other uncontrolled medical comorbidities; 4) treated with definitive CRT, all patients completed treatment plan; 5) adequate hematological, liver, and renal function; and 6) without any active infections or autoimmune diseases. This study was approved by the medical ethics committee of Shandong Cancer Hospital and Institute; all participants provided written informed consent.

\section{Treatment protocols}

All the patients in this study received platinum-based bimodal cytotoxic drug chemotherapy concurrent with radiation therapy but without targeted therapy. All radiation treatments were delivered as either 3D-CRT or intensity-modulated radiation therapy (IMRT) with standard fractionation (ie, 1.8/2.0 Gy fractions once daily for 5 days/week). Radiation was delivered by high-energy (6 or $15 \mathrm{MV}$ ) linear accelerators. Patients were treated with a total dose of 50-64 Gy administered over 25-32 fractions. Chemotherapy began on Day 1 concurrent with the initial radiation treatments, and the regimens included 2 cycles of 5-fluorouracil and cisplatin. 5-Fluorouracil $\left(700 \mathrm{mg} / \mathrm{m}^{2}\right)$ was administered intravenously (iv) on Days $1-3$, and cisplatin $\left(25 \mathrm{mg} / \mathrm{m}^{2}\right)$ was administered by iv infusion in $500 \mathrm{~mL}$ of either normal saline or dextrose on Days 1-3.

\section{Blood sample analysis}

All routine blood tests were performed within 1 week prior to treatment initiation and were collected in tubes containing ethylenediaminetetraacetic acid (EDTA). The white blood cell (WBC), neutrophil, platelet, lymphocyte, and monocyte counts were extracted in a retrospective fashion from the medical records. LMR was defined as dividing the lymphocyte count by the monocyte count using pretreatment blood test results.

\section{Assessment of response and follow-up}

Evaluation of the primary tumor response to treatment was performed by using CT and esophagography 2-4 weeks after completion of CRT. This assessment was based on comparisons of the pre- and posttreatment imaging study findings and was performed using Response Evaluation Criteria in Solid Tumors (RECIST) criteria; patients were classified in 1 of 4 response categories. A CR was defined as no evidence of disease and tumor marker measurements 
within normal ranges for at least 2 weeks. Partial response (PR) was defined as a decrease in the lesion as measured bidimensionally by at least $30 \%$ with no signs of either new lesions or progression of any existing lesions. Progressive disease (PD) was defined as an increase of at least $20 \%$ in a lesion as measured bidimensionally, the appearance of any new lesions, or a previously eradicated lesion reappearing. Stable disease (SD) was defined as a tumor response that did not fulfill the PR criteria but exceeded the PD criteria.

Either a contrast-enhanced CT or a PET-CT scan was performed to evaluate the tumor and node status every 3 months for the first 2 years and every 6 months thereafter for all patients; also, an endoscopy and barium meal were conducted every 6 months. The last follow-up date was September 10, 2015.

\section{Statistical analysis}

Analyses were performed using the Statistical Package for Social Science (SPSS, Version 22.0; SPSS Inc, Chicago, IL, USA) program, and a 2 -sided $P$-value $<0.05$ implied statistical significance. The associations between the LMR and clinicopathological parameters (ie, gender, age, drinking history, smoking history, tumor location, clinical tumor stage [cT] status clinical node stage [cN] status, tumor stage, and tumor response) were assessed by using the chi-squared test. Logistic regression model was used to determine the clinical tumor response predictors. Progression-free survival (PFS) was defined as the period from the date of therapy initiation to the date of detected disease progression. Overall survival (OS) was defined as the period from the date of therapy initiation to either the date of an individual's death due to any cause or the date of the last follow-up. The association of each marker with PFS and OS was analyzed using Kaplan-Meier plots and the log-rank test, and the associated 95\% CIs were calculated. Multivariate analyses were conducted using the Cox proportional hazards model with the variables with a $P$-value of $<0.05$ in the univariate analysis and were constructed with the backward stepwise method. All tests were two sided, and $P<0.05$ was considered statistically significant.

\section{Results}

\section{Patient characteristics}

A total of 162 patients were enrolled in the study, including $127(78.4 \%)$ males and 35 (21.6\%) females, with a median age of 63 years (range: $38-70$ years). All the included patients received definitive CRT, and most of the patients received additional courses of chemotherapy ranging from 2 to 4 cycles. The median follow-up was 23.3 months (range: 8-43.7 months). During this period, 113 (69.8\%) patients experienced progression, and $89(54 \%)$ patients died. Of the 162 tumor cases, 51 (31.5\%) cases were located in either the upper or middle esophagus, and 111 (68.5\%) cases were located in the lower region, including the gastroesophageal junction $(9,5.6 \%)$; additionally, most of the patients had stage III disease $(129,79.6 \%)$, whereas 33 (20.4\%) had stage II disease.

The median value of the pretreatment LMR was 4.02 (interquartile range [IQR]: 1.77-3.11). We chose this as the cutoff value for the LMR and categorized patients into the high or low group; thus, 81 patients had a detected LMR less than or equal to 4.02, and 81 patients had an LMR >4.02 (Table 1).

\section{Relationship between the LMR and clinicopathological characteristics}

The relationship between the LMR and clinicopathological characteristics is shown in Table 1. None of the clinicopathological characteristics (including gender, drinking history, smoking history, tumor location, and node status) could

Table I Relationship between pretreatment LMR and clinicopathological parameters in patients with ESCC who received definitive chemoradiotherapy

\begin{tabular}{|c|c|c|c|c|}
\hline $\begin{array}{l}\text { Clinicopathological } \\
\text { parameters }\end{array}$ & $\begin{array}{l}\text { Cases } \\
(n=162)\end{array}$ & LMR $\leq 4.02$ & LMR $>4.02$ & $P$-value \\
\hline Gender & & & & 1.000 \\
\hline Female & $35(21.6 \%)$ & 18 & 17 & \\
\hline Male & $127(78.4 \%)$ & 63 & 64 & \\
\hline Age, years & & & & 0.024 \\
\hline$\leq 60$ & $63(38.9 \%)$ & 24 & 39 & \\
\hline$>60$ & $99(61.1 \%)$ & 57 & 42 & \\
\hline Drinking history & & & & 0.090 \\
\hline Ever & $5 \mathrm{I}(3 \mathrm{I} .5 \%)$ & 31 & 20 & \\
\hline Never & III (68.5\%) & 50 & 61 & \\
\hline Smoking history & & & & 1.000 \\
\hline Ever & 97 (59.9\%) & 49 & 48 & \\
\hline Never & 65 (40.1\%) & 32 & 33 & \\
\hline Tumor location & & & & 0.735 \\
\hline Upper, mid & $5 \mathrm{I}(3 \mathrm{I} .5 \%)$ & 24 & 27 & \\
\hline Lower, GE junction & III (68.5\%) & 57 & 54 & \\
\hline cT status & & & & 0.228 \\
\hline $\mathrm{TI}-3$ & II 4 (70.4\%) & 53 & 61 & \\
\hline $\mathrm{T} 4$ & 48 (29.6\%) & 28 & 20 & \\
\hline $\mathrm{cN}$ status & & & & 0.176 \\
\hline No & $34(21.0 \%)$ & 13 & 21 & \\
\hline $\mathrm{NI}-3$ & I 28 (79.0\%) & 68 & 60 & \\
\hline Tumor stage & & & & 0.018 \\
\hline II & 33 (20.4\%) & 10 & 23 & \\
\hline III & 129 (79.6\%) & 71 & 58 & \\
\hline Tumor response & & & & $<0.001$ \\
\hline $\mathrm{CR}$ & $48(29.6 \%)$ & 12 & 36 & \\
\hline Not CR & II 4 (70.4\%) & 69 & 45 & \\
\hline
\end{tabular}

Note: Bold values are significant $(P<0.05)$.

Abbreviations: CR, complete response; ESCC, esophageal squamous cell carcinoma; GE, gastroesophageal; LMR, lymphocyte-monocyte ratio; cT, clinical tumor stage; cN, clinical node stage. 
predict significant differences between the high and low LMR groups. However, an increased LMR was significantly correlated with age $(P=0.024)$, tumor stage $(P=0.018)$, and clinical tumor response $(P<0.001)$.

\section{Univariate and multivariate analyses of the clinical tumor response}

As shown in Table 2, the number of patients in the CR and non-CR group was 48 (29.6\%) and 114 (70.4\%), respectively. Tumor-related parameters, including the $\mathrm{cT}$ status $(P=0.053)$, $\mathrm{cN}$ status ( $P=0.523)$, and tumor stage $(P=0.279)$, showed no significant differences between the $\mathrm{CR}$ and non-CR groups. Among the hematological characteristics, there was a trend of decreased neutrophil counts $(P=0.187)$ and monocyte counts $(P=0.052)$ in the $\mathrm{CR}$ group, but these decreases were not significant. Conversely, the platelet count level $(P=0.029)$ was often lower in the CR group. However, the lymphocyte count $(P=0.018)$, the percentage of lymphocytes in the WBC count $(P=0.004)$, and the LMR $(P<0.001)$ were significantly higher in the $\mathrm{CR}$ group compared with the values in the non-CR group. According to the RECIST criteria, there were $12(12 / 81)$ patients with CR in the low LMR group vs $36(36 / 81)$ patients with CR in the high LMR group $(P<0.001)$.

Only the significant variables from the univariate analysis (platelet count, lymphocyte count, the percentage of lymphocytes, and the LMR) were included in the subsequent multivariate logistic regression models (Table 3). Considering

Table 2 Univariate and multivariate analyses for tumor response (CR and non-CR)

\begin{tabular}{|c|c|c|c|}
\hline \multirow[t]{2}{*}{ Parameters } & \multicolumn{2}{|c|}{ Tumor response } & \multirow{2}{*}{$\begin{array}{l}\text { Univariate } \\
\text { analysis } \\
P \text {-value }\end{array}$} \\
\hline & $\begin{array}{l}\text { CR } \\
(n=48)\end{array}$ & $\begin{array}{l}\text { Non-CR } \\
(n=1 \mid 4)\end{array}$ & \\
\hline cT status & & & 0.053 \\
\hline TI-3 & 39 & 75 & \\
\hline $\mathrm{T} 4$ & 9 & 39 & \\
\hline $\mathrm{cN}$ status & & & 0.523 \\
\hline No & 8 & 24 & \\
\hline $\mathrm{NI}-3$ & 40 & 90 & \\
\hline Tumor stage & & & 0.279 \\
\hline II & 12 & 20 & \\
\hline III & 36 & 94 & \\
\hline Neutrophils $\left(\times 10^{9} / \mathrm{L}\right)$ & $3.97 \pm 1.27$ & $4.30 \pm 1.52$ & 0.187 \\
\hline Platelets $\left(\times 10^{9} / \mathrm{L}\right)$ & $207.75 \pm 55.34$ & $230.21 \pm 59.42$ & 0.029 \\
\hline Monocytes $\left(\times 10^{9} / \mathrm{L}\right)$ & $0.46 \pm 0.17$ & $0.52 \pm 0.200$ & 0.052 \\
\hline Lymphocytes $\left(\times 10^{9} / \mathrm{L}\right)$ & $2.00 \pm 0.84$ & $1.69 \pm 0.58$ & 0.018 \\
\hline \%Lymphocytes in WBC & $29.83 \% \pm 8.43 \%$ & $26.01 \% \pm 6.95 \%$ & 0.004 \\
\hline LMR & $4.89 \pm 1.17$ & $3.87 \pm 1.29$ & $<0.001$ \\
\hline
\end{tabular}

Note: Bold values are significant $(P<0.05)$.

Abbreviations: $\mathrm{CR}$, complete response; $\mathrm{cT}$, clinical tumor stage; $\mathrm{cN}$, clinical node stage; LMR, lymphocyte-monocyte ratio; WBC, white blood cell.
Table 3 Multivariate analysis for tumor response (CR and non-CR)

\begin{tabular}{|c|c|c|c|c|}
\hline \multirow[t]{2}{*}{ Parameters } & \multicolumn{2}{|l|}{ Model I } & \multicolumn{2}{|l|}{ Model 2} \\
\hline & HR (95\% Cl) & $P$-value & HR (95\% Cl) & $P$-value \\
\hline Platelets & $1.01(1.00-1.02)$ & 0.051 & $1.01(1.00-1.01)$ & 0.160 \\
\hline Lymphocytes & $0.59(0.31-1.13)$ & 0.112 & - & - \\
\hline $\begin{array}{l}\text { \%Lymphocytes } \\
\text { in WBC }\end{array}$ & - & - & $0.97(0.92-1.03)$ & 0.327 \\
\hline LMR & $0.63(0.46-0.87)$ & 0.006 & $0.60(0.43-0.82)$ & 0.002 \\
\hline
\end{tabular}

Note: Bold values are significant $(P<0.05)$.

Abbreviations: $C R$, complete response; HR, hazard ratio; LMR, lymphocytemonocyte ratio; WBC, white blood cell.

the multicollinearity between the lymphocyte count and the percentage of lymphocytes, two different models were used (Table 3). Among the included variables in both models, only an elevated LMR was a significant prognostic factor for a good clinical tumor response $(P<0.05)$.

\section{Prognostic variables for PFS and OS}

The median PFS was 11.6 months, and the median OS was 23.3 months. As shown in Figure 1, the Kaplan-Meier curves for PFS and OS revealed that, compared to patients in the low LMR group, patients in the high LMR group had a longer PFS $(P<0.001$, Figure 1A) and OS $(P<0.001$, Figure 1B)

In the univariate analysis, in addition to LMR, the cT stage status (PFS, $P<0.001$; OS, $P=0.006$ ), tumor stage (PFS, $P=0.013$; OS, $P=0.017$ ), and clinical tumor response (PFS, $P<0.001$; OS, $P<0.001$ ) were also significantly associated with PFS and OS (Table 4).

A multivariate Cox proportional hazards model was used to assess independent prognostic factors. In the multivariate analysis, the cT status $(P=0.007$, hazard ratio $[\mathrm{HR}]=0.58 ; 95 \%$ CI: $0.39-0.86)$, clinical tumor response $(P=0.004, \mathrm{HR}=0.51$; 95\% CI: $0.32-0.81)$, and LMR $(P<0.001, \mathrm{HR}=2.17 ; 95 \%$ CI: 1.46-3.23) remained as significant prognostic indicators of PFS, whereas the tumor stage ( $P=0.041, \mathrm{HR}=0.52 ; 95 \%$ CI: 0.28-0.97), clinical tumor response $(P=0.016, \mathrm{HR}=0.52$; $95 \%$ CI: $0.31-0.88)$, and LMR $(P=0.002$, HR $=2.02 ; 95 \% \mathrm{CI}$ : 1.29-3.18) had a significant relationship with OS (Table 5). Overall, a good clinical tumor response and high LMR were favorable independent predictive factors for PFS and OS (all $P<0.05$ ).

\section{Discussion}

In this study, we retrospectively analyzed a consecutive cohort of ESCC patients who underwent definitive CRT and found that an elevated pretreatment LMR predicts not only a good clinical tumor response but also a lower risk of recurrence and cancer-related death. To the best of our knowledge, there are few studies that have focused on the role of the 

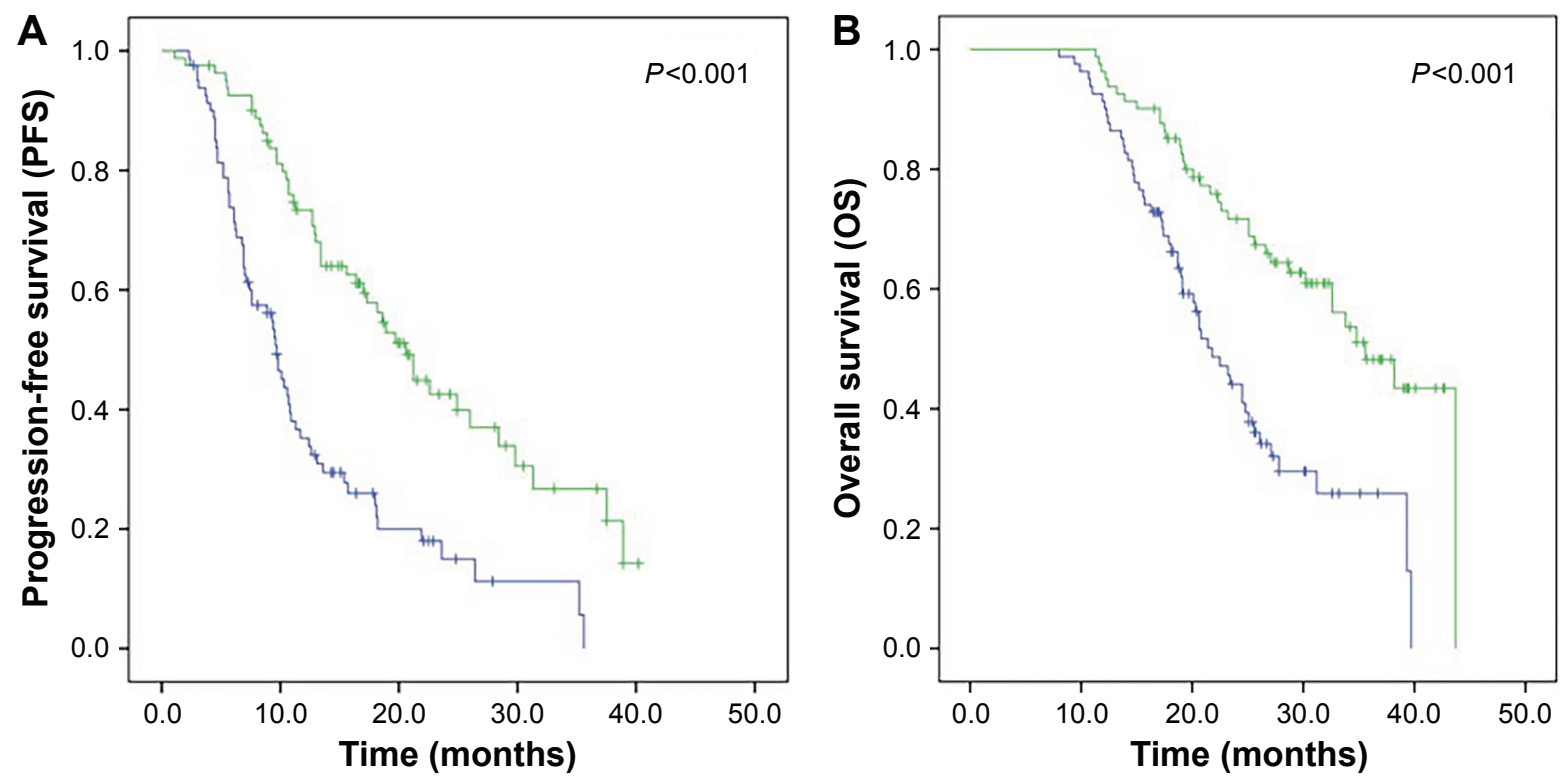

$$
\begin{aligned}
& \text { LMR } \\
& \neg \text { LMR } \leq 4.02-\neg \text { LMR }>4.02+\text { LMR } \leq 4.02 \text { censored }+ \text { LMR }>4.02 \text { censored }
\end{aligned}
$$

Figure I Kaplan-Meier curves for patients with low and high LMR.

Notes: (A) Kaplan-Meier curves for PFS and (B) Kaplan-Meier curves for OS.

Abbreviation: LMR, lymphocyte-monocyte ratio.

pretreatment LMR in predicting outcomes of ESCC patients, especially those who underwent definitive CRT.

Accumulating evidence has demonstrated a strong link between system inflammation and cancer; the peripheral inflammatory cells, including neutrophils, lymphocytes, and monocytes represented significant markers of systematic inflammatory response. Lymphocytes can not only suppress tumor cell proliferation and migration ${ }^{21}$ but also eliminate residual tumor cell and micrometastases. ${ }^{22}$ Lymphocytes play an important role in promoting antitumor immunity; lymphopenia might weaken the efficacy of the immune system. In this study, we observed that a high count $(P=0.018)$ and percentage $(P=0.004)$ of circulating lymphocytes were associated with a good clinical tumor response, which is consistent with the results of other studies. ${ }^{23,24}$ Kitayama et $\mathrm{al}^{23}$ found that patients with locally advanced rectal cancer who had high circulating lymphocyte percentages before radiotherapy (RT) showed better clinical response to RT than their matched counterparts with lower circulating lymphocyte percentages. Thus, a relatively high number or percentage of circulating lymphocytes might indicate a more robust antitumor immune response.

Different from the role of lymphocytes, tumor-associated macrophages (TAMs) exert a protumorigenic effect by

\begin{tabular}{|c|c|c|c|c|}
\hline \multirow[t]{2}{*}{ Parameter } & \multicolumn{2}{|l|}{ PFS } & \multicolumn{2}{|l|}{ os } \\
\hline & HR $(95 \% \mathrm{Cl})$ & $P$-value & HR $(95 \%$ Cl) & $P$-value \\
\hline Gender (female/male) & $1.20(0.16-0.21)$ & 0.347 & $1.83(0.22-0.30)$ & 0.264 \\
\hline Age $(\leq 60 />60)$ & $1.76(0.15-0.22)$ & 0.333 & $1.61(0.26-0.32)$ & 0.818 \\
\hline Drinking history (ever/never) & I.3I (0.57-0.74) & $0.70 \mathrm{I}$ & I.53 (0.29-0.4I) & 0.816 \\
\hline Smoking history (ever/never) & $1.34(0.16-0.21)$ & 0.698 & $1.21(0.26-0.31)$ & 0.548 \\
\hline Tumor location (upper, mid/low, GE) & $1.35(0.68-1.57)$ & 0.867 & $1.42(0.28-0.35)$ & 0.596 \\
\hline cT status $(\mathrm{TI}-3 / \mathrm{T} 4)$ & $1.33(0.17-0.22)$ & $<0.001$ & $1.21(0.28-0.33)$ & 0.006 \\
\hline cN status (negative/positive) & $2.03(0.15-0.22)$ & 0.730 & $2.26(0.25-0.34)$ & 0.873 \\
\hline Tumor stage (II, III) & $2.27(0.19-0.28)$ & 0.013 & $2.20(0.29-0.38)$ & 0.017 \\
\hline Response (CR/not CR) & $1.98(0.21-0.29)$ & $<\mathbf{0 . 0 0 1}$ & $1.59(0.30-0.37)$ & $<0.001$ \\
\hline $\operatorname{LMR}(\leq 4.02 />4.02)$ & $1.21(0.10-0.15)$ & $<0.001$ & $1.25(0.22-0.27)$ & $<\mathbf{0 . 0 0 1}$ \\
\hline
\end{tabular}

Table 4 Univariate analysis of factors associated with PFS and OS

Note: Bold values are significant $(P<0.05)$.

Abbreviations: CR, complete response; cT, clinical tumor stage; cN, clinical node stage; GE, gastroesophageal; HR, hazard ratio; LMR, lymphocyte-monocyte ratio; PFS, progress-free survival; OS, overall survival. 
Table 5 Multivariate analysis of factors associated with PFS and OS

\begin{tabular}{|c|c|c|c|c|}
\hline \multirow[t]{2}{*}{ Parameters } & \multicolumn{2}{|l|}{ PFS } & \multicolumn{2}{|l|}{ OS } \\
\hline & HR (95\% Cl) & $P$-value & HR (95\% Cl) & $P$-value \\
\hline cT status & $0.58(0.39-0.86)$ & 0.007 & $0.70(0.44-1.12)$ & 0.137 \\
\hline Tumor stage & $0.70(0.4 I-\mid .2 I)$ & 0.200 & $0.52(0.28-0.97)$ & $0.04 I$ \\
\hline Tumor response & $0.5 \mathrm{I}(0.32-0.8 \mathrm{I})$ & 0.004 & $0.52(0.31-0.88)$ & 0.016 \\
\hline LMR & $2.17(1.46-3.23)$ & $<\mathbf{0 . 0 0 I}$ & $2.02(1.29-3.18)$ & 0.002 \\
\hline
\end{tabular}

Note: Bold values are significant $(P<0.05)$.

Abbreviations: $c T$, clinical tumor stage; HR, hazard ratio; LMR, lymphocytemonocyte ratio; PFS, progress-free survival; OS, overall survival.

accelerating tumor progression via secretion of growth factors and cytokines such as tissue-type plasminogen activator (t-PA), platelet-derived growth factor (PDGF), interleukin (IL)-6, and several matrix metalloproteinases (MMPs), all of which promote tumor angiogenesis and reduced immunosurveillance. ${ }^{25,26}$ TAMs originating from circulating monocytes are stimulated by the tumor-derived chemotactic factors and are recruited to the tumor site. ${ }^{27}$ Thus, circulating monocytes could substitute for TAMs in peripheral blood to reflect tumor burden. Koh et al ${ }^{28}$ reported a significant positive correlation between the TAMs and monocyte percentages in peripheral blood and a negative correlation between the TAM count and LMR. We discovered that circulating monocyte counts were associated with tumor response, albeit not significantly $(P=0.052)$; this was similar to the results of a previous study. ${ }^{29}$

Because the LMR is indicative of the relationship between antitumor and protumor immune activities, it is reasonable to speculate that an elevated LMR can be interpreted to mean that lymphocyte-mediated antitumor immune activities are stronger than monocyte-mediated protumor immune activities, which favors a better clinical tumor response. Go et a ${ }^{30}$ analyzed data from 171 patients with small-cell lung cancer and found that the ratio (98.3\%) of the response (CR or PR) was higher in the high LMR group than the low LMR group $(P=0.02)$. In our study, we observed similar results: there were more CR patients in the high LMR group $(36 / 48, P<0.001)$, and the LMR value was higher in the CR group than that in the non-CR group ( $4.89 \pm 1.17$ vs $3.87 \pm 1.29, P<0.001)$, which supports the correlation between elevated LMR and better tumor response in ESCC patients who received definitive CRT.

With regard to recurrence and survival, Nishijima et al performed a meta-analysis of 29 studies of solid tumors and showed that the HR of low preoperative LMR was 1.73 (95\% CI: $1.55-1.93 ; P<0.001)$ for OS and 1.56 (95\% CI: $1.31-1.86 ; P<0.001)$ for DFS. ${ }^{20}$ There was also a study that focused on the correlation between LMR and survival in ESCC patients. ${ }^{31}$ The author included 218 patients who had pathologically confirmed ESCC and underwent radical surgery. The author reported that patients with an LMR $\leq 2.57$ had shorter DFS and OS than patients with an LMR $>2.57$. However, Huang and Feng ${ }^{32}$ inferred that only 5 -year cancer-specific survival was associated with LMR. Notably, both reports focused on patients who underwent esophagectomy without considering patients who underwent definitive CRT. In this study, in patients with an LMR $\leq 4.02$, the median PFS and OS were 9.3 months and 19.7 months, respectively; in patients whose $\mathrm{LMR}>4.02$, the values were 16.6 months and 27.6 months, respectively. The results of this study demonstrated that an elevated LMR independently predicted PFS $(P<0.001)$ and OS $(P=0.002)$ in locally advanced ESCC patients who received definitive CRT.

The major limitations of our study are the retrospective analysis, single-center design, and small sample size. In addition, there are other drawbacks to this study. First, we chose the median value of the LMR as the cutoff value to investigate whether any trends existed. Although this approach may minimize a false-positive result, it is unclear whether a different cutoff value or threshold would serve as a better predictor of tumor recurrence and survival in ESCC patients. Second, the TNM stage of the patients is only the clinical stage, and although we used EUS and contrast-enhanced CT to determine the staging, these approaches may restrict the ability to evaluate the prognosis of ESCC compared with that of pathological staging. The same problem also exists in assessing the tumor response by using RECIST criteria. However, it is highly difficult to obtain pathological information for nonoperable patients. Therefore, additional large-scale, multicenter prospective trials are needed to confirm the reproducibility of these results in a heterogeneous population.

\section{Conclusion}

An elevated pretreatment LMR indicates a good clinical tumor response for patients with locally advanced ESCC who received definitive CRT; furthermore, this parameter is also considered an indicator to predict a low risk of recurrence and improved survival. The abovementioned findings may aid clinicians to identify patients who are more susceptible to recurrence and more sensitive to CRT such that they can receive more aggressive treatment.

\section{Acknowledgment}

This study was funded by the Natural Science Foundation of China (NSFC grant number 81672995) and by the Science and Technology Program of Shandong Province (Grant number 2016GSF201133). 


\section{Disclosure}

The authors report no conflicts of interest in this work.

\section{References}

1. Jemal A, Bray F, Center MM, Ferlay J, Ward E, Forman D. Global cancer statistics. CA Cancer J Clin. 2011;61(2):69-90.

2. Zeng H, Zheng R, Guo Y, et al. Cancer survival in China, 2003-2005: a population-based study. Int J Cancer. 2015;136(8):1921-1930.

3. Thomas P, Doddoli C, Lienne P, et al. Changing patterns and surgical results in adenocarcinoma of the oesophagus. Br J Surg. 1997;84(1): $119-125$.

4. Cooper JS, Guo MD, Herskovic A, et al. Chemoradiotherapy of locally advanced esophageal cancer: long-term follow-up of a prospective randomized trial (RTOG 85-01). Radiation Therapy Oncology Group. JAMA. 1999;281(17):1623-1627.

5. Minsky BD, Pajak TF, Ginsberg RJ, et al. INT 0123 (Radiation Therapy Oncology Group 94-05) phase III trial of combined-modality therapy for esophageal cancer: high-dose versus standard-dose radiation therapy. $J$ Clin Oncol. 2002;20(5):1167-1174.

6. Higuchi K, Koizumi W, Tanabe S, et al. Current management of esophageal squamous-cell carcinoma in Japan and other countries. Gastrointest Cancer Res. 2009;3(4):153-161.

7. Meng X, Wang J, Sun X, et al. Cetuximab in combination with chemoradiotherapy in Chinese patients with non-resectable, locally advanced esophageal squamous cell carcinoma: a prospective, multicenter phase II trail. Radiother Oncol. 2013;109(2):275-280.

8. Rice TW, Blackstone EH, Rusch VW. 7th edition of the AJCC Cancer Staging Manual: esophagus and esophagogastric junction. Ann Surg Oncol. 2010;17(7):1721-1724.

9. Dunn GP, Old LJ, Schreiber RD. The immunobiology of cancer immunosurveillance and immunoediting. Immunity. 2004;21(2):137-148.

10. Xie X, Luo KJ, Hu Y, Wang JY, Chen J. Prognostic value of preoperative platelet-lymphocyte and neutrophil-lymphocyte ratio in patients undergoing surgery for esophageal squamous cell cancer. Dis Esophagus. 2016;29(1):79-85.

11. Man YG, Stojadinovic A, Mason J, et al. Tumor-infiltrating immune cells promoting tumor invasion and metastasis: existing theories. $J$ Cancer. 2013;4(1):84-95.

12. Formenti SC, Demaria S. Effects of chemoradiation on tumorhost interactions: the immunologic side. J Clin Oncol. 2008;26(9): $1562-1563$.

13. DeNardo DG, Johansson M, Coussens LM. Immune cells as mediators of solid tumor metastasis. Cancer Metastasis Rev. 2008;27(1):11-18.

14. Solinas G, Marchesi F, Garlanda C, Mantovani A, Allavena P. Inflammation-mediated promotion of invasion and metastasis. Cancer Metastasis Rev. 2010;29(2):243-248.

15. Jaiswal M, LaRusso NF, Burgart LJ, Gores GJ. Inflammatory cytokines induce DNA damage and inhibit DNA repair in cholangiocarcinoma cells by a nitric oxide-dependent mechanism. Cancer Res. 2000;60(1): 184-190.

16. Song YJ, Wang LX, Hong YQ, et al. Lymphocyte to monocyte ratio is associated with response to first-line platinum-based chemotherapy and prognosis of early-stage non-small cell lung cancer patients. Tumour Biol.2016; 37(4):5285-5293.
17. Fujiwara Y, Misawa T, Shiba H, et al. Postoperative peripheral absolute blood lymphocyte-to-monocyte ratio predicts therapeutic outcome after pancreatic resection in patients with pancreatic adenocarcinoma. Anticancer Res. 2014;34(9):5163-5168.

18. Rochet NM, Kottschade LA, Grotz TE, Porrata LF, Markovic SN. The prognostic role of the preoperative absolute lymphocyte count and absolute monocyte count in patients with resected advanced melanoma. Am J Clin Oncol. 2015;38(3):252-258.

19. Li J, Jiang R, Liu WS, et al. A large cohort study reveals the association of elevated peripheral blood lymphocyte-to-monocyte ratio with favorable prognosis in nasopharyngeal carcinoma. PLoS One. 2013;8(12):e83069.

20. Nishijima TF, Muss HB, Shachar SS, Tamura K, Takamatsu Y. Prognostic value of lymphocyte-to-monocyte ratio in patients with solid tumors: a systematic review and meta-analysis. Cancer Treat Rev. 2015; 41(10):971-978.

21. Bastid J, Bonnefoy N, Eliaou JF, Bensussan A. Lymphocyte-derived interleukin-17A adds another brick in the wall of inflammation-induced breast carcinogenesis. Oncoimmunology. 2014;3:e28273.

22. Nazir T, Islam A, Omer MO, Mustafa M. Lymphocytopenia; induced by vinorelbine, doxorubicin and cisplatin in human cancer patients. Breast Dis. 2015;35(1):1-4.

23. Kitayama J, Yasuda K, Kawai K, Sunami E, Nagawa H. Circulating lymphocyte is an important determinant of the effectiveness of preoperative radiotherapy in advanced rectal cancer. BMC Cancer. 2011;11:64.

24. Kitayama J, Yasuda K, Kawai K, Sunami E, Nagawa H. Circulating lymphocyte number has a positive association with tumor response in neoadjuvant chemoradiotherapy for advanced rectal cancer. Radiat Oncol. 2010;5:47.

25. Krstic J, Santibanez JF. Transforming growth factor-beta and matrix metalloproteinases: functional interactions in tumor stroma-infiltrating myeloid cells. ScientificWorldJournal. 2014;2014:521754.

26. Schaue D, Comin-Anduix B, Ribas A, et al. T-cell responses to survivin in cancer patients undergoing radiation therapy. Clin Cancer Res. 2008; 14(15):4883-4890.

27. Chanmee T, Ontong P, Konno K, Itano N. Tumor-associated macrophages as major players in the tumor microenvironment. Cancers (Basel). 2014; 6(3):1670-1690.

28. Koh YW, Kang HJ, Park C, et al. The ratio of the absolute lymphocyte count to the absolute monocyte count is associated with prognosis in Hodgkin's lymphoma: correlation with tumor-associated macrophages. Oncologist. 2012;17(6):871-880.

29. Vetsika EK, Koinis F, Gioulbasani M, et al. A circulating subpopulation of monocytic myeloid-derived suppressor cells as an independent prognostic/predictive factor in untreated non-small lung cancer patients. J Immunol Res. 2014;2014:659294.

30. Go SI, Kim RB, Song HN, et al. Prognostic significance of the lymphocyte-to-monocyte ratio in patients with small cell lung cancer. Med Oncol. 2014;31(12):323.

31. Han LH, Jia YB, Song QX, Wang JB, Wang NN, Cheng YF. Prognostic significance of preoperative lymphocyte-monocyte ratio in patients with resectable esophageal squamous cell carcinoma. Asian Pac J Cancer Prev. 2015;16(6):2245-2250.

32. Huang Y, Feng JF. Low preoperative lymphocyte to monocyte ratio predicts poor cancer-specific survival in patients with esophageal squamous cell carcinoma. Onco Targets Ther. 2015;8:137-145.
OncoTargets and Therapy

\section{Publish your work in this journal}

OncoTargets and Therapy is an international, peer-reviewed, open access journal focusing on the pathological basis of all cancers, potential targets for therapy and treatment protocols employed to improve the management of cancer patients. The journal also focuses on the impact of management programs and new therapeutic agents and protocols on

\section{Dovepress}

patient perspectives such as quality of life, adherence and satisfaction. The manuscript management system is completely online and includes a very quick and fair peer-review system, which is all easy to use. Visit http://www.dovepress.com/testimonials.php to read real quotes from published authors. 